\title{
Editorial
}

\section{Recent Trends in Computational and Theoretical Aspects in Differential and Difference Equations}

\author{
Ram Jiwari, ${ }^{1}$ Mehmet Emir Koksal, ${ }^{2}$ and Haydar Akca ${ }^{3}$ \\ ${ }^{1}$ Department of Mathematics, Indian Institute of Technology Roorkee, Roorkee 247667, India \\ ${ }^{2}$ Department of Mathematics, Ondokuz Mayis University, 55139 Samsun, Turkey \\ ${ }^{3}$ Department of Applied Sciences and Mathematics, College of Arts and Sciences, Abu Dhabi University, \\ P.O. Box 59911, Abu Dhabi, UAE \\ Correspondence should be addressed to Ram Jiwari; ramlmaths@gmail.com
}

Received 6 July 2017; Accepted 9 July 2017; Published 11 September 2017

Copyright (C) 2017 Ram Jiwari et al. This is an open access article distributed under the Creative Commons Attribution License, which permits unrestricted use, distribution, and reproduction in any medium, provided the original work is properly cited.

Differential and difference equations are used for modeling, solving, and discussing many problems arising in engineering and natural sciences. Therefore, analysis of analytical and numerical solutions to such equations is crucial for applications. It is important to develop new theories and methods, as well as modify the well-known techniques.

The main propose of this special issue was to attract high quality contributions and highlight significant recent developments on the subject of differential and difference equations. The focus of this special issue was also originated with numerical methods for solving differential equations and modelling and/or considering engineering problems involving differential and difference equations. Below we give a brief survey of the content of this special issue.

The special issue succeeded in bringing together a number of papers on various branches of the theory and applications of both differential and difference equations. One of the papers in the issue is on the convergence of the solutions of the bistable reaction-diffusion equations in two-dimensional spaces to a pair of diverging traveling curved fronts. Another one focuses on developing a mixed discontinuous Galerkin scheme for the following control constrained optimal control problem governed by a transient convection-diffusion equation. One paper studies the stability analysis of the periodic solutions of some kind of predator-prey dynamical systems. There is also an important paper on finding exact solution of nonlinear sixth-order solitary wave equation using the improved generalized tanh-coth method. Finally, as a novel application of differential equations in system and control theory, the investigation of the inverse commutativity conditions for second-order linear continuous time-varying systems is studied in another paper.

\section{Acknowledgments}

Finally, the guest editors of the present special issue wish to take this opportunity to thank all authors for their contributions, which have provided a range of in-depth overviews of the most relevant topics in differential and difference equations. They are also grateful to distinguished scientists who served as referees for the submitted manuscripts. It is hoped that the papers published in the special issue will enhance and stimulate future studies and developments in the field.

Ram Jiwari Mehmet Emir Koksal Haydar Akca 


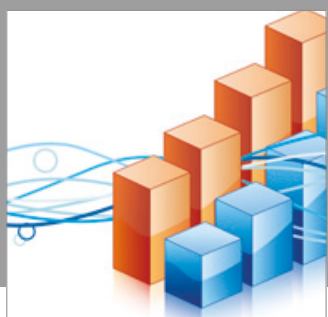

Advances in

Operations Research

vatersals

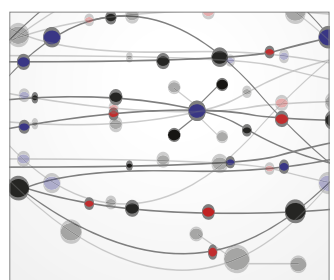

\section{The Scientific} World Journal
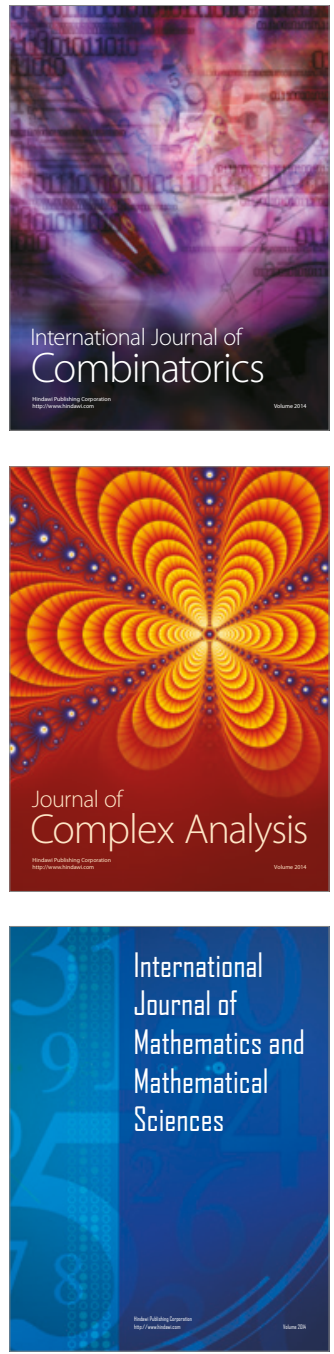
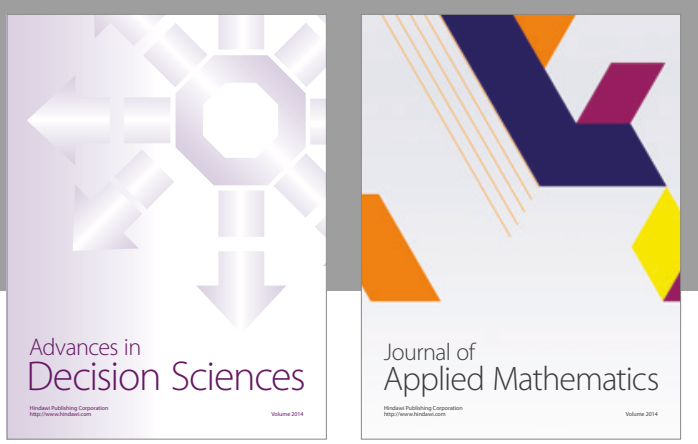

Algebra

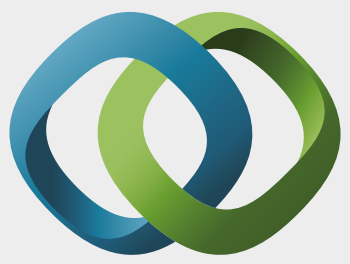

\section{Hindawi}

Submit your manuscripts at

https://www.hindawi.com
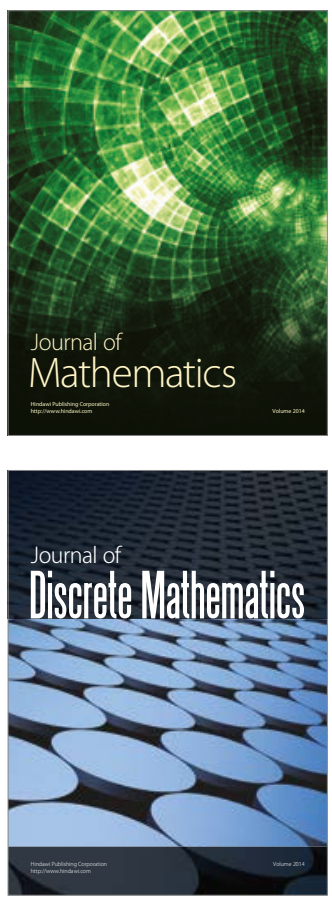

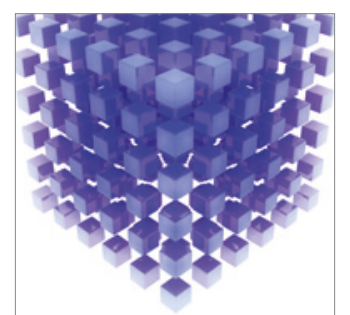

Mathematical Problems in Engineering
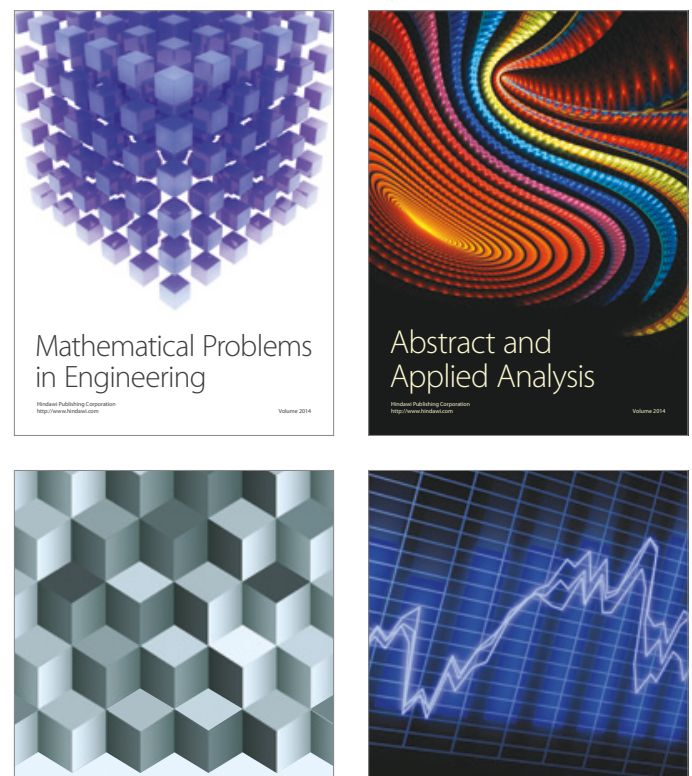

Journal of

Function Spaces

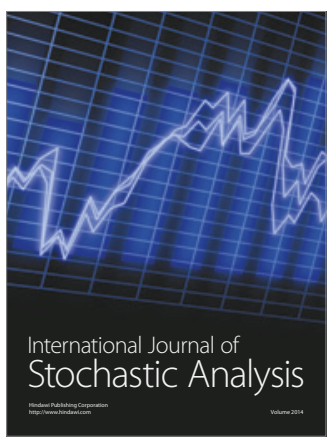

Probability and Statistics
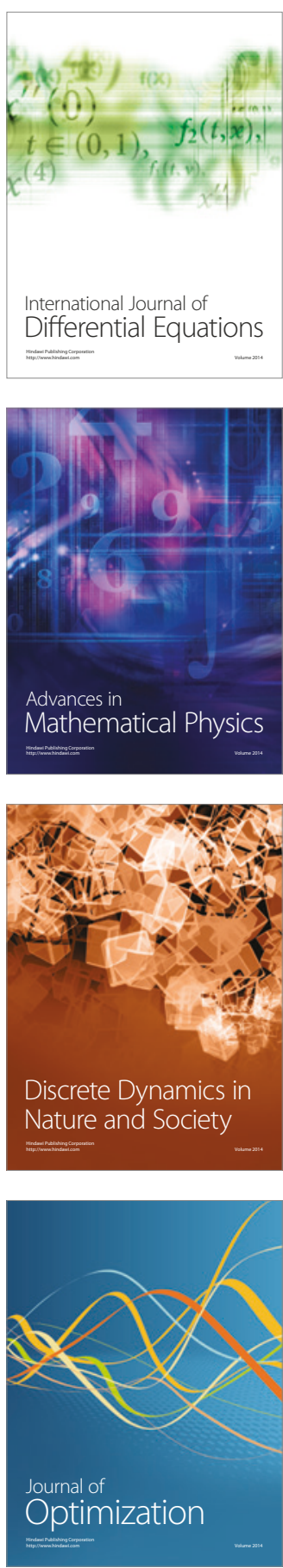